\title{
Surface plasmon polaritons on deep, narrow-ridged rectangular gratings
}

\author{
M. R. Gadsdon, * I. R. Hooper, A. P. Hibbins, and J. R. Sambles \\ School of Physics, University of Exeter, Exeter, Devon, EX4 4QL, United Kingdom \\ *Corresponding author: m.r.gadsdon@exeter.ac.uk
}

Received January 6, 2009; revised April 16, 2009; accepted April 17, 2009; posted April 27, 2009 (Doc. ID 105719); published May 19, 2009

\begin{abstract}
The dispersion diagrams of surface plasmon polaritons have been calculated for rectangular gratings, with very narrow wires, of varying depths. For gratings with a moderate height a family of vertical-standing-wave resonances may be excited, which consist of surface plasmons, oscillating on either vertical surface, coupling together through the metal wires. These modes evolve similarly to the manner in which shallow-grating surface-plasmon dispersion curves evolve into cavity modes in the grooves of the structure. However, on further increase in grating height these vertical standing waves evolve into a second resonant feature, which is independent of yet further increases in height. This new mode is shown to be equivalent to the resonances found on infinite multilayer metal/dielectric structures illuminated at normal incidence. (C) 2009 Optical Society of America

OCIS codes: $240.6680,050.6624,230.4170$.
\end{abstract}

\section{INTRODUCTION}

The primary differences between the electromagnetic response of a thick planar metal film and a thick metal film that has shallow periodic perturbations on its surface have long been understood as the emergence of diffractive orders and the excitation of surface plasmon polaritons (SPPs) [1-3].

It had been thought that SPPs could not be excited in the subwavelength regime, where the periodicity of the surface relief perturbation (grating) is significantly less than the wavelength of the incident radiation. However, it has been shown that surface plasmons may be excited in the zero-order region of the spectrum, as they evolve to form cavity modes in the grooves of the structure [4-6]. Further work has also examined the nature of these cavity modes on gratings whose ridges are sufficiently narrow that the surface plasmon modes on each vertical surface may also interact through the metal ridges, as well as across the grooves $[7,8]$. These cavity modes are essentially the same as the TEM cavity mode in a single groove, except that their evolution from normal surface plasmon modes, due to the periodicity of the structure, means that there are some subtle differences in their dispersion curves from the well-known TEM mode.

There has also been significant research into planar multilayered metal/dielectric stacks, where the transmission coefficient through such stacks may be several orders of magnitude larger than that through a single layer of the metal film [9].

The spectral response of such structures comprises of a series of bandpass regions, where transmissivity is high and reflectivity low, separated by a series of photonic band gaps where the opposite is true. It has been demonstrated that, near either band edge, there is a large enhancement of the field intensity due to localization effects $[10,11]$. Near the high-frequency band edge of the lowest frequency bandpass region, the electromagnetic field is predominantly confined to the dielectric regions in a manner similar to a stack of Fabry-Perot cavities, while at the low-frequency band edge, the fields are more concentrated in the metal regions $[10,12,13]$.

Recently, we have explained the formation of the bandpass regions as being due to the matching of the tangential electric and magnetic fields associated with standing cavity modes in the dielectric, which may have either a $\cos$ or a $\sin$ wave character, and evanescent standing modes in the metal, which may have either a cosh or a sinh distribution function [10].

It was shown that the low-frequency band edge of the lowest-frequency bandpass region is formed by the matching of cosh fields in the metal layers and cos standing waves in the cavities, with adjacent cavities oscillating in phase. The high-frequency edge is then formed by the matching of sinh fields in the metal layers with cos standing waves in the dielectric layers, with adjacent cavities oscillating out of phase-much like a series of FabryPerot cavities. The boundary conditions imposed by the evanescent standing modes may significantly modify the effective cavity length that the standing waves in the cavities experience. The sinh fields result in an effective cavity length that is very similar to the physical cavity length, such that the stack behaves as a series of FabryPerot cavities. The cosh standing waves are able to give a much larger effective cavity length than the physical cavity thickness, this significantly redshifts the lowfrequency edge of the normal single-cavity Fabry-Perot resonance, thereby opening up a large bandpass region.

The next bandpass region is formed in the same way, except that the fields inside the cavities have a $\sin$ wave function. Subsequent bandpass regions are then the result of cavity harmonics matching to either cosh or sinh fields in the metal layers. Of course, this is only exactly 
true for structures with an infinite number of layers, as it is only then that the fields inside the metal and dielectric layers can form profiles with exactly cosh or sinh, and $\cos$ or $\sin$ functions, respectively. In addition, it has been shown that the multilayer structures also support SPPs when illuminated in an attenuated total reflection configuration $[14,15]$.

The present work examines the electromagnetic response of a surface relief grating, with a rectangular cross-section profile, as the ridges of the grating are made very thin and then made very high. It is shown that vertical standing waves, that are coupled across the metal ridges rather than the grooves, exist when the ridges are very narrow. As the grating height is increased, these modes evolve into the same modes that exist on metal/ dielectric multilayered stacks.

\section{THEORETICAL MODEL}

All of the modelling in the present work utilizes the rigorous coupled-wave analysis technique, also called the Fourier modal method [16]. In this technique the system is split into three regions, as in Fig. 1. The permittivity profile in the $x$-direction is approximated as a Floquet series expansion. In each region, the electromagnetic wave is described as the superposition of "upward" and "downward" waves in the $y$-direction. Each of these waves is then described as a Rayleigh expansion of eigenmodes, each of which is also expanded according to the Floquet series expansion associated with the approximation of the permittivity profile. Maxwell's equations are explicity solved in each region according to the description above of the electromagnetic fields. The resultant solutions are then used to match the electromagnetic boundary conditions, at the interface between each region, using the numerically stable scattering matrix [17].

Figure 1 gives a schematic representation of the model used in this work. Region 2 is the grating layer consisting of silver and air with grating height $h$, period $d$, and mark-to-space ratio $f$. Region 1 , the incidence medium is

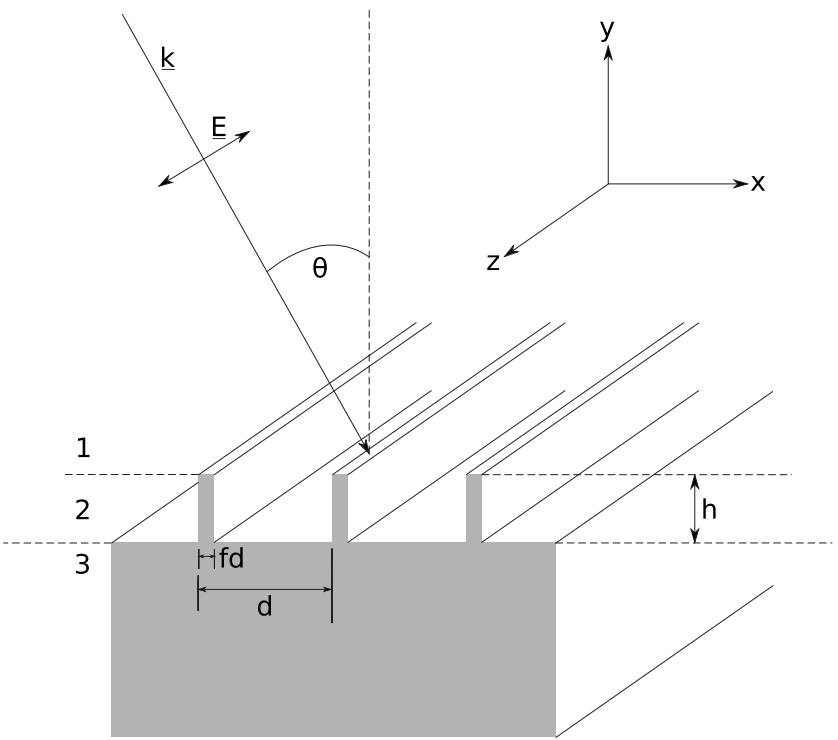

Fig. 1. Schematic representation of the theoretical model. air, and region 3 is silver such that the structure is a continuous surface relief grating. The incident radiation $k$ is at an angle $\theta$ to the interface, normal with the incident plane containing the grating vector. It is linearly polarized with the $E$-field perpendicular to the long axis of the grating wires, TM (in the plane of incidence).

A practical determination of the convergence of the code can be specified by the absolute difference between the approximate and exact (very high truncation order $M$ ) values. Thus the code can be determined to have converged once the difference between the values obtained for $M$ and $M+1$ are lower than an arbitrary value, chosen as

$$
\frac{\left|\delta_{M+1}-\delta_{M}\right|}{\left|\delta_{M+1}\right|}<0.001
$$

The difference between the exact and approximate values will be different for every single point of a given plot because it will be dependent not only on the geometry of the structure, but also on the permittivity and wavelength. Evidently then, a different truncation order investigation will be required for every point to ensure convergence has been achieved.

Of course, it would be very inefficient to establish these criteria for every single point of every single plot. The compromise, for the sake of practicability, is to implement a low-resolution plot over the parameter space of interest, at a moderate truncation order, which will reveal the approximate location of the resonance(s) of interest. Then, the convergence criteria are determined, according to Eq. (1), for the maxima or minima of the resonance(s). Finally, the full resolution plot is calculated for the parameter space using a truncation order at least as high as the minimum that is required according to the previously determined convergence criteria. This process was implemented for every plot given in the present work, and it typically resulted in truncation orders $M \geqslant 80$.

The permittivity of silver is specified by the Drude model, using the parameters $\omega_{p}=1.32 \times 10^{16} \mathrm{~s}^{-1}$ and $\tau_{D}$ $=1.45 \times 10^{-14} s$, taken from Nash and Sambles [18]. One needs to be aware that the permittivities in the case of a thin wire, as in region 2, may be different from those of an infinite film. It has been discussed elsewhere that the classical macroscopic approach of electrical permittivity is adequate for particle dimensions as small as $2 \mathrm{~nm}$ [19]. As a bulk metal is reduced in thickness towards this value, the mean free path of the electrons decreases as the electron surface scattering becomes more important. However, it has been shown that, for such silver wires as studied here, only the imaginary part of the permittivity increases slightly [19]. Thus, it is the present authors' belief that the underlying physics presented here is sound and that the changes in permittivity will only result in a slight broadening and reduction in magnitude of the resonances.

\section{RESULTS}

In order to examine the optical response of a rectangular grating with a large amplitude and very narrow wires, we begin with a grating with thick wires, which supports cav- 
ity modes and then vary the width of the wires. This evolution is shown in Fig. 2, where the reflection efficiency of a grating of height $h=350 \mathrm{~nm}$ and period $d=200 \mathrm{~nm}$, that is illuminated at $\theta=4.89^{\circ}$, is presented, while the markto-space ratio is varied in the range $0.05 \leqslant f \leqslant 0.95$. The wavelength range is $370 \mathrm{~nm} \leqslant \lambda \leqslant 850 \mathrm{~nm}$, which equates to a frequency range of $2.22 \times 10^{15} \mathrm{rad} \cdot \mathrm{s}^{-1} \leqslant \omega \leqslant 5.1$ $\times 10^{15} \mathrm{rad} \cdot \mathrm{s}^{-1}$. The slight dotted effect is due to a lower resolution for $f$ and $d$ than for $\omega$. Note that the dotted lines indicate the location of the subsequent field plots shown in Figs. 3 and 5.

At the high $f$ values of Fig. 2 there is a family of modes (including the lowest-frequency mode for $0.5 \leqslant f \leqslant 0.9$ ), which increase in frequency as $f$ reduces, that is as the wires narrow and the grooves broaden. This family of modes exists even for optically thick wires; therefore, they cannot be a family of surface plasmons coupled across the wires. For cavity modes in a single groove in a perfect conductor, it has been shown that end effects lead to a decrease in frequency as the groove is broadened [20]. However, it has been discussed elsewhere [21] that, for very narrow grooves in a finite conductor, as the groove is broadened there is an increase in frequency of the cavity mode due to the finite conductivity, and that in the optical regime, this effect dominates [22]. It is therefore expected that cavity modes, on the structure presented here, will increase in frequency as the grooves are broadened, and furthermore, that the resonance will broaden and diminish. This is clearly the case for the family of modes at high $f$ values, identifying these as a family of cavity modes.

To confirm this hypothesis, the $\left|H_{z}\right|$-fields associated with the two lowest frequency resonances observable at $f=0.93$ are presented in Fig. 3. Figure 3(a) gives the $\left|H_{z}\right|$-fields for the higher-frequency reflection minimum in Fig. 2 at $f=0.93$ and $\omega=3.77 \times 10^{15} \mathrm{rad} \cdot \mathrm{s}^{-1}$; Fig. $3(\mathrm{~b})$ gives the $\left|H_{z}\right|$-fields for the lowest reflection minimum at $f$ $=0.93$ and $\omega=2.90 \times 10^{15} \mathrm{rad} \cdot \mathrm{s}^{-1}$. The black line indicates

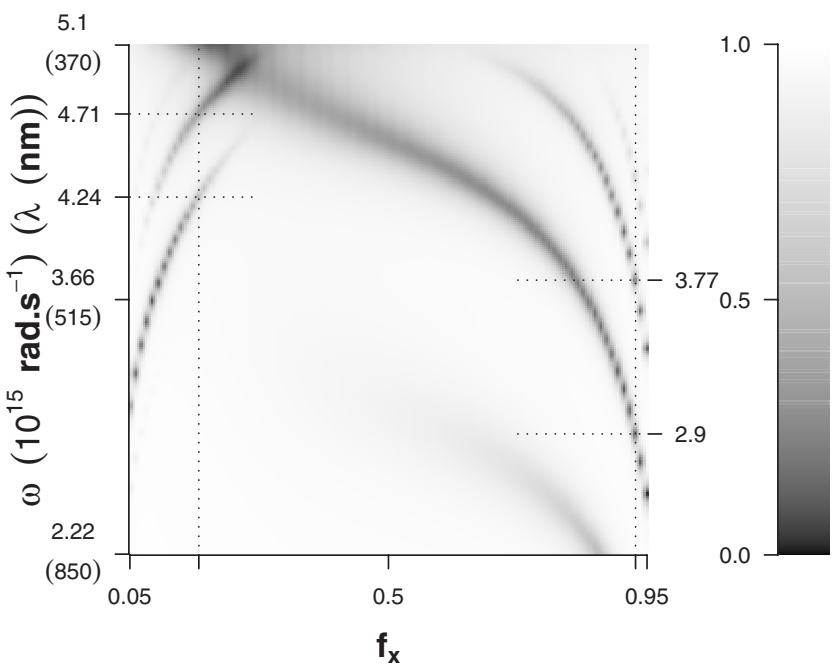

Fig. 2. Reflection efficiency response of the grating as a function of both the incident frequency and $f$. The fixed grating parameters are $d=200 \mathrm{~nm}, h=350 \mathrm{~nm}$, and $\theta=4.89^{\circ}$. The wavelength range is $370 \mathrm{~nm} \leqslant \lambda \leqslant 850 \mathrm{~nm}$, which equates to a frequency range of $2.22 \times 10^{15} \mathrm{rad} \cdot \mathrm{s}^{-1} \leqslant \omega \leqslant 5.1 \times 10^{15} \mathrm{rad} \cdot \mathrm{s}^{-1}$. $f$ is in the range $0.05 \leqslant f \leqslant 0.95$. The dotted lines indicate the location of the subsequent field plots (Figs. 3 and 5).
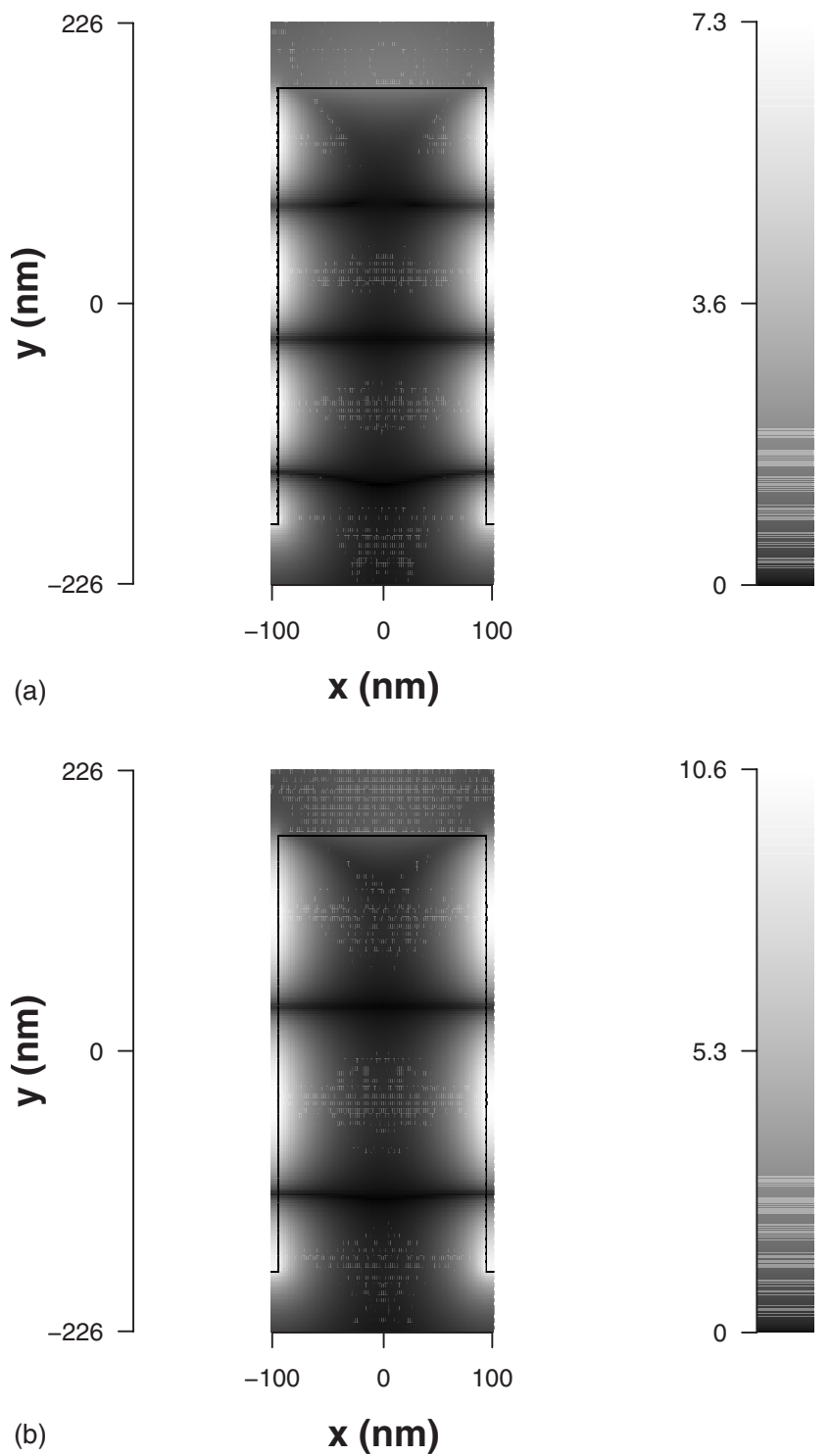

(b)

\section{$\mathbf{x}(\mathbf{n m})$}

Fig. 3. $\left|H_{z}\right|$ for the two lowest-frequency reflection minima in Fig. 2 for $f=0.93$. The black line indicates the location of the surface of the grating.

the location of the surface of the grating. Note that the scales are different and that the lower-order mode has a larger maximum field enhancement.

Clearly, the two reflection minima are adjacent harmonics of the fundamental cavity mode resonance that exists in the grooves of the structure when the wires are optically thick and the grooves are narrow. Furthermore, examining the dependence of this family of modes on the grating height, and examining their dispersion diagrams as the height is varied, shows that they evolve from normal surface plasmon modes and, subsequently, they behave in exactly the same way as the cavity modes discussed in a similar investigation into surface relief gratings with a Gaussian cross-section [6].

Returning to Fig. 2, we now consider the family of resonances that occur for low values of $f$ and which decrease in frequency as the wires are narrowed (and the grooves are broadened). Figure 4 presents a similar investigation to that given in Fig. 2, except that the period is allowed to 

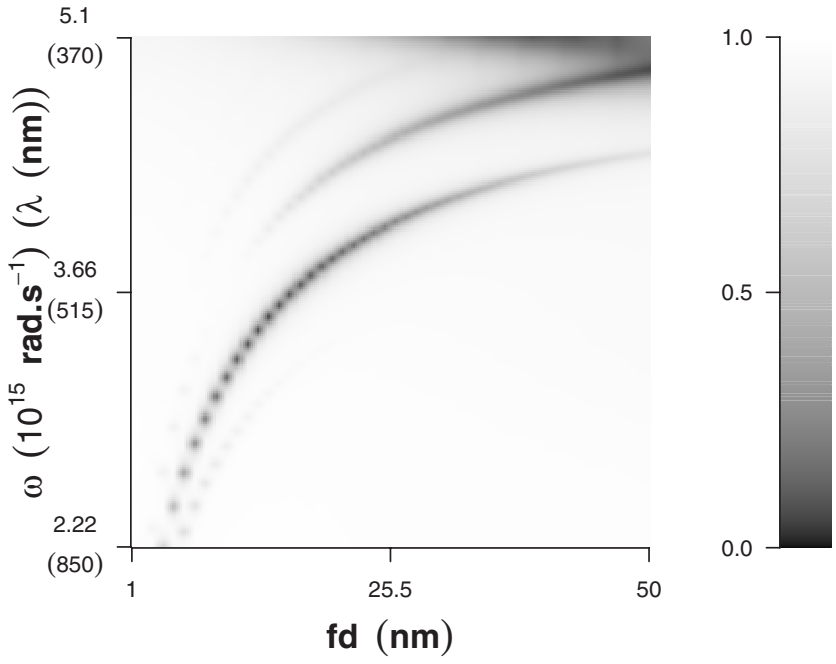

Fig. 4. Reflection efficiency response of the grating as a function of both the incident frequency and wire width, $f d$. The fixed grating parameters are $(1-f) d=166 \mathrm{~nm}, h=350 \mathrm{~nm}$, and $\theta=4.89^{\circ}$. The frequency range is the same as in Fig. 2 . The wire width is in the range $1 \mathrm{~nm} \leqslant f d \leqslant 50 \mathrm{~nm}$.

vary such that the groove width is held constant, at (1 $-f) d=166 \mathrm{~nm}$, as the wire width is varied in the range $1 \mathrm{~nm} \leqslant f d \leqslant 50 \mathrm{~nm}$.

When the wire width is varied for a constant groove width, the family of resonances behaves in an almost identical way to when the groove width is allowed to vary. This indicates that the groove width has little or no influence on the resonance, and hence, the resonance is not a cavity mode.

In order to examine the nature of the thin wire resonances, Fig. 5(a) gives the $H_{z}$-fields for the higherfrequency reflection minimum in Fig. 2 at $f=0.17$ and $\omega=4.71 \times 10^{15} \mathrm{rad} \cdot \mathrm{s}^{-1}$; Fig. 5(b) gives the $H_{z}$-fields for the lowest reflection minimum at $f=0.17$ and $\omega=4.24$ $\times 10^{15} \mathrm{rad} \cdot \mathrm{s}^{-1}$. The black line indicates the location of the surface of the grating. If the phase of the incident radiation is assumed to be $\phi=0$, these instantaneous fields are plotted at a phase of $\phi=\pi / 2$ - the phase at which maximum field enhancement occurs.

Figure 5 indicates that the two resonances are also harmonics of a vertical standing wave resonance. However, the resonance is not a standing wave associated with the cavities of the structure, but with the wires. That is, surface plasmons on the walls of the wires are now coupled together through the wires rather than across the grooves. It is noted that the penetration depth of the fields into silver (the value at which their magnitude falls to $1 / e$ ) in the frequency range of Fig. 2 is approximately $25 \mathrm{~nm}$. This correlates with the fact that the highest value of $f$ at which these resonances occur is seen to be $f \simeq 0.3$, which gives a wire width of $\sim 49.8 \mathrm{~nm}$-approximately twice the decay length, which is expected because the resonances occur on either side of the wire.

These resonances are similar to the resonances found previously on thin Gaussian ridges [7,8]. The instantaneous fields also show that the coupling across the wires is asymmetric, that is, $H_{z}$ goes through a zero inside the metal. This explains why these modes cannot be excited at normal incidence. The symmetry of the H-fields re-

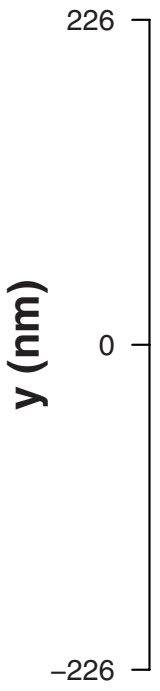

(a)
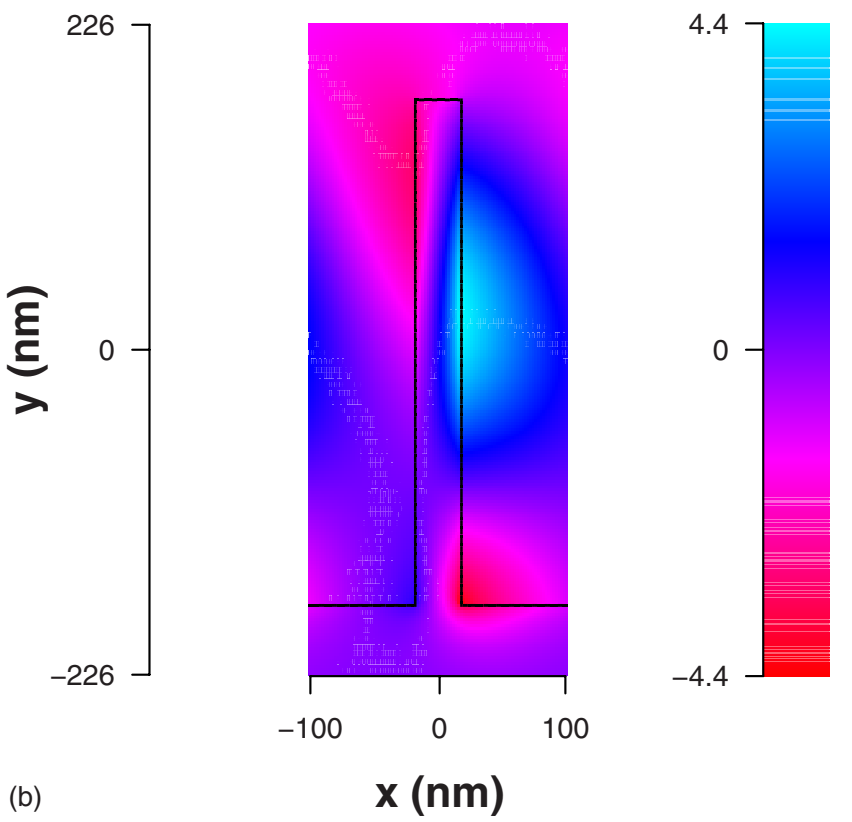

(b)
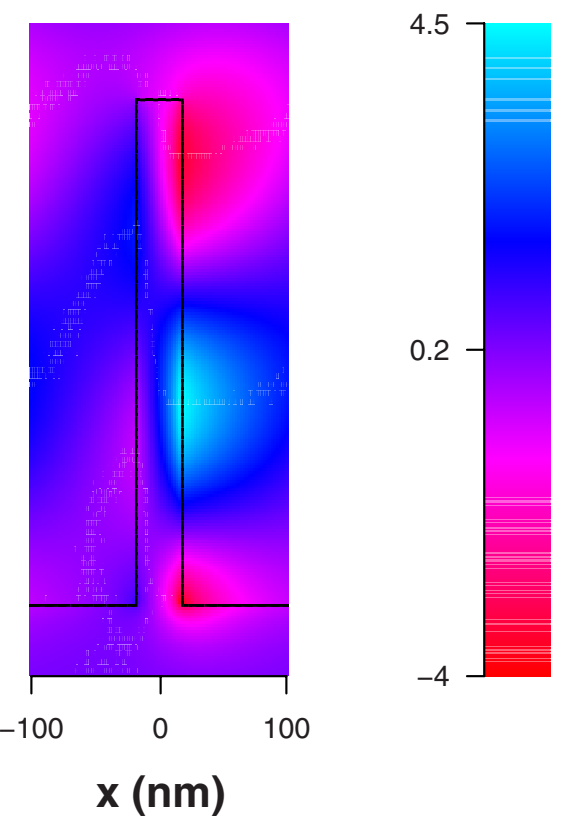

Fig. 5. (Color online) $H_{z}$ for the two lowest-frequency reflection minima in Fig. 2 for $f=0.17$. Both fields are plotted at a phase of $\phi=\pi / 2$ relative to the incident radiation at a phase $\phi=0$. The black line indicates the location of the surface of the grating.

quires that the E-field on opposite sides of the groove point in exactly opposite directions; therefore, this mode cannot be coupled to at normal incidence because, at any instant in time, it is impossible for the incident E-field to match this field distribution. Away from normal incidence this symmetry is broken, and the incident E-field can match components of the resonant E-field distribution and thus excite the mode.

The reduction in frequency as the wires become thinner is expected from the behavior of coupled surface plasmons on a thin semi-infinite film. On such a film, the evanescent field of the normal surface plasmon excited on one interface is able to excite a second surface plasmon on the opposite interface. These two modes are degenerate if the 
adjacent dielectrics are the same. As they become more strongly coupled, the normal surface plasmon dispersion curve undergoes a splitting into a high- and a lowfrequency branch, each of which increase and decrease in frequency, respectively, as the film is made thinner [23]. The high-frequency branch is associated with a symmetric distribution of $H_{z}$ across the film, while the lowfrequency branch has an asymmetric distribution [24]. The fraction of the fields of the symmetric mode that exist inside the metal, where absorption dominates, reduces as the film is made thinner; therefore, absorption losses decrease and the resonances' propagation length increases-the long-range, coupled SPP. The opposite is true for the asymmetric resonance-the short-range, coupled SPP [25]. Therefore, as the coupling across the wires is asymmetric, it is expected that the resonance will redshift in frequency as the wire becomes thinner.

This analysis also corroborates the discussed increase in frequency of the cavity mode resonance as the groove is widened. Surface plasmons coupled across a dielectric between two metals behave in the opposite sense to the situation described above. It is well known for cavity modes that the coupling is symmetric across the gap; therefore, because the symmetric/asymmetric modes behave in the opposite sense, it is expected that the mode will increase in frequency as the groove is broadened.

The nature of the low $f$ standing waves may now be investigated further. We first examine the spectral dependence of these modes on grating height to ascertain if they have the $1 / h$ frequency response that would be expected for vertical standing waves, while taking into account the possibility of dispersion curve anticrossing that has been shown to arise for cavity resonances on deep gratings [6]. We then proceed with a similar investigation as carried out by Hooper and Sambles [6], observing the dispersion diagrams of the structure as the grating height is varied, in order to examine the evolution of these modes from normal SPPs.

Figure 6 gives the dependence of the standing waves on

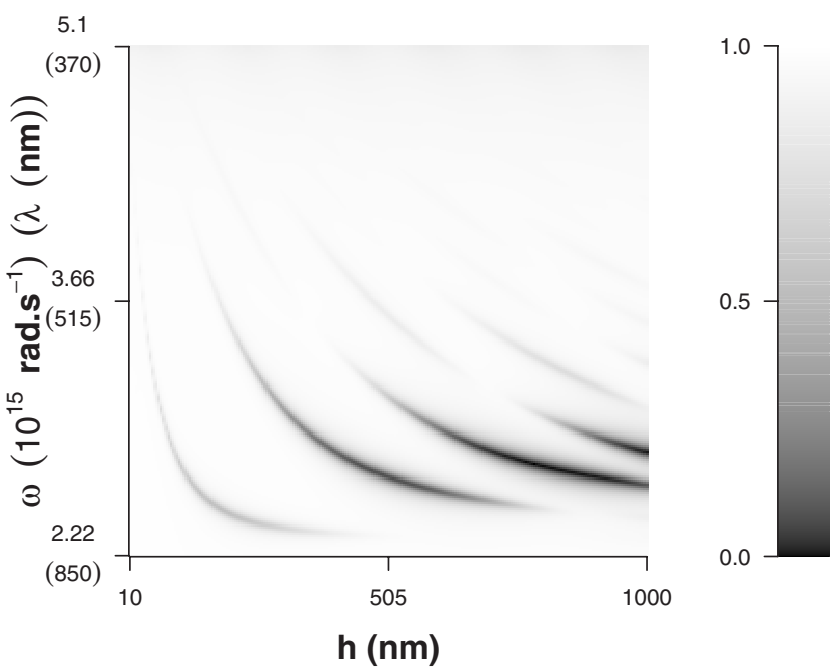

Fig. 6. Reflection efficiency response of the grating as a function of both the incident frequency and grating height, $h$. The fixed grating parameters are $(1-f) d=225 \mathrm{~nm}, f d=10 \mathrm{~nm}$, and $\theta$ $=4.89^{\circ}$. The frequency range is the same as in Fig. 2 . The height is in the range $10 \mathrm{~nm} \leqslant h \leqslant 1000 \mathrm{~nm}$. the grating height in the range $10 \mathrm{~nm} \leqslant h \leqslant 1000 \mathrm{~nm}$. The first feature that can be observed is that the standing waves do not have a true $1 / h$ dependence. At low grating heights there is an approximately $1 / h$ dependence; however, this dependence does not continue to zero frequency, as $h \rightarrow \infty$, because there appears to be a finite limit frequency that all of the modes tend towards, with the higher-frequency modes tending to this limit more slowly at increasingly larger values of grating height. The field plots of Fig. 5 show that, in the vertical direction, there is definite harmonic behavior between adjacent resonances, indicating that they have a vertical standing wave character. This fact, combined with their approximate $1 / h$ dependence for small values of $h$ (Fig. 6), shows that the resonances behave as vertical standing waves at low grating height. However, they cannot be true standing waves for all grating heights because they do not tend to zero frequency as the height tends to infinity.

To address this question, it is now important to look at the evolution of the standing waves from normal surface plasmon modes. To achieve this aim, Figs. 7(a), 7(b), 8(a), and $8(\mathrm{~b})$, give the dispersion diagrams of a grating with wires $6.5 \mathrm{~nm}$ wide, grooves $166 \mathrm{~nm}$ wide, and varying in heights of $h=40,80,160,320 \mathrm{~nm}$, respectively. The dispersion diagram is calculated by determining the poles of the global scattering matrix, $S$, by summing over all its elements. The dotted lines indicate the light line and the first-order diffracted line.

In Figure 7(a) there are two flat-banded modes that exist before the light line. If the wires were thick and very shallow, it would be expected that the surface plasmon dispersion would not be significantly perturbed. Therefore, the surface plasmon modes would be expected at very high frequencies - the first band gap would be expected at $\lambda=345 \mathrm{~nm}$. However, because the wires are very thin, and at $40 \mathrm{~nm}$ not very shallow, they are causing significant perturbation to the surface plasmon propagation and hence their dispersion curves. This is a result of a combination of the wire amplitude no longer being negligible, and hence perturbing the SPP propagation, and then these perturbed modes interacting through the thin metal wires and result in the opening of large and flat band gaps. Thus, the $k_{g}, 2 k_{g} \ldots, q k_{g}$ scattered surface plasmon modes have been brought down significantly in frequency and have also flattened. This can also be observed in the nonradiative region where the unscattered mode has been brought down in frequency by a large amount. Figures 7(b) and 8 show that this modification of the surface plasmon modes continues to a greater extent, as the grating height is increased further, so that many flat modes may be observed when $h=320 \mathrm{~nm}$ [Fig. 8(b)].

This evolution is almost identical to the evolution of normal surface plasmon modes into cavity modes on gratings with broad wires [6]. Therefore, this evolution together with previous field plots (Fig. 5) and the dependence on grating height (Fig. 6), confirms that, at low grating heights, the modes act as vertical standing waves, which are coupled across the thin wires and thus are localized to each wire, as seen by the flat-banded behavior. However, as shown in the previous work on gratings with thick wires, if true cavity modes are formed, then this evolution continues such that the flat bands are displaced 

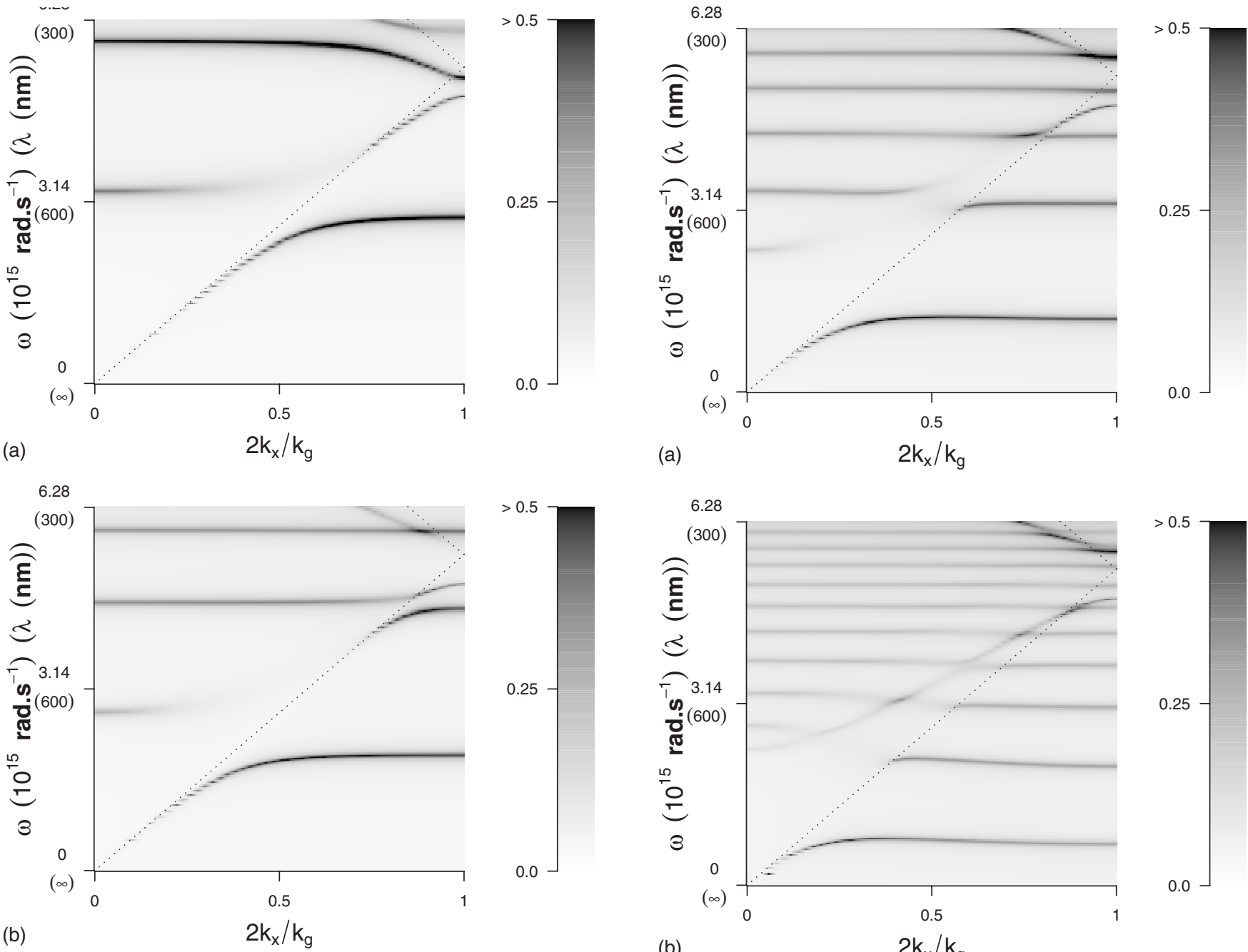

Fig. 7. Poles of the scattering matrix of a silver rectangular grating as a function of both the incident frequency and in-plane momentum for increasing $h$. The fixed grating parameters are $d=172.5 \mathrm{~nm}, f d=6.5 \mathrm{~nm}$. The frequency is in the range $0<\omega$ $\leqslant 6.28 \times 10^{15} \mathrm{rad} \cdot \mathrm{s}^{-1}$, giving a wavelength range $\infty>\lambda \geqslant 300 \mathrm{~nm}$, and the in-plane momentum is in the range $0 \leqslant 2 k_{x} / k_{g} \leqslant 1$. The dotted lines indicate the light line and the first-order diffracted line.

down in frequency continuously, as the grating height is increased, and they tend to zero frequency. This is not the case for standing waves coupled across the wires, as there is a clear finite frequency limit to which all of the modes tend. Beyond approximately $h=300 \mathrm{~nm}$, the lowestfrequency mode has reached this limit and has formed into a new type of mode, which is no longer flat-banded, and appears to increase in frequency, toward a different limit, as $2 k_{x} / k_{g} \rightarrow 1$. In Fig. 8(b) this is the $s$-shaped mode that starts at $\omega \approx 2.35 \times 10^{15} \mathrm{rad} \cdot \mathrm{s}^{-1}$, when $2 k_{x} / k_{g}=0$, and increases in frequency to $4.96 \times 10^{15} \mathrm{rad} \cdot \mathrm{s}^{-1}$ when $2 k_{x} / k_{g}=1$. As the grating height increases further, the higher-order scattered modes shift down in frequency and also tend to this new mode. This behavior shows that, at low grating heights, the normal surface plasmon modes evolve into vertical standing modes that are coupled across the wires of the grating. However, as the grating height is increased further, the standing modes then evolve into a new type of resonance.

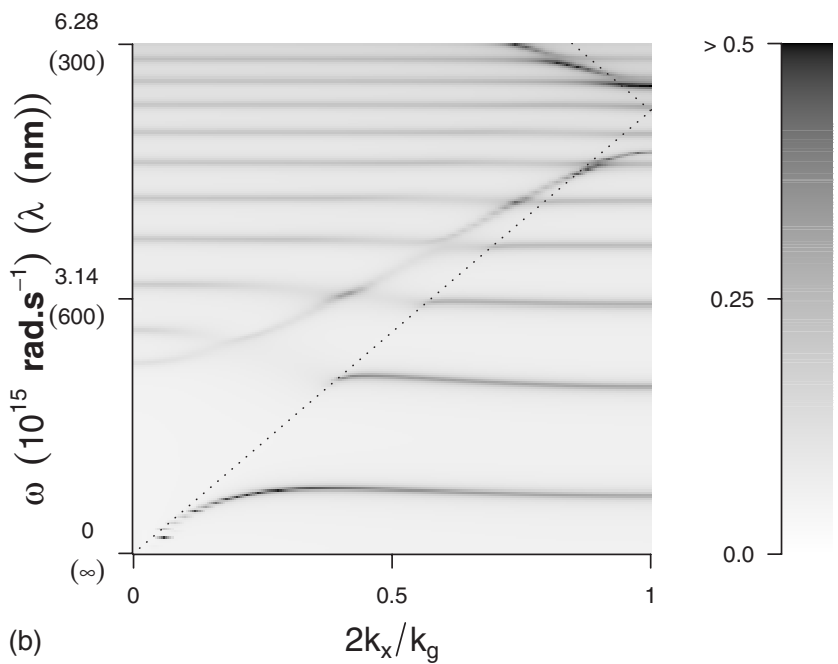

Fig. 8. Poles of the scattering matrix of a silver rectangular grating as a function of both the incident frequency and in-plane momentum for increasing $h$. The fixed grating parameters are $d=172.5 \mathrm{~nm}, f d=6.5 \mathrm{~nm}$. The frequency and in-plane momentum ranges are the same as in Fig. 7.

Of course, the reduction in frequency of the dispersion of the normal SPP modes, as the grating is made deeper, can also be understood through previous work on spoof plasmons [26-29]. This red-shift can be considered as the structure of the metal inducing plasmoniclike behavior well away from the true plasma frequency of silver. In the referenced work, the spoof plasmon modes occur where the light line experiences anticrossings with the nondispersive cavity resonances (which can be considered as having formed as a result of the self-coupling of SPPs in the grooves of the structure [6]). In other words, the redshift of the SPP occurs due to the self-coupling of SPPs in the grooves forming cavity resonances, and where these nondispersive modes anticross with the light line spoof plasmons are formed. Similar behavior can be seen in Figs. 7(a) and 7(b); however, the subtle difference in the present work is that the presence of spoof plasmons is due to the anticrossing of the light line with nondispersive standing-wave resonances in the wire, not in the cavities. For the larger gratings heights, where the new mode has evolved, as can be seen in Figs. 7(c) and 7(d), the anticrossing is clearly much more complicated. 
This new resonance is independent of further increases in grating height, this implies that, beyond a certain height, the structure is effectively acting as a multilayer metal/dielectric structure with each layer being (effectively) infinitely wide, and the whole structure consisting of an infinite number of layers at least to the lowest-order standing wave. The grating height must be increased further to act this way for the higher-order standing waves. It is possible to investigate whether the grating is indeed behaving as an infinite multilayer structure, to the lowest-order mode, by comparing the response of this mode to the theory, which has recently been presented describing the behavior of truly infinite multilayer stacks [10].

Figure 9 is a plot showing the dependence of the reflection efficiency response of a multilayer structure comprising of ten silver layers of thickness, $b$, in the range $1 \mathrm{~nm} \leqslant b \leqslant 50 \mathrm{~nm}$, separated by nine $166 \mathrm{~nm}$ air layers. In [10], it was shown that only the (negative) real part of the permittivity of the silver is important in the formation of the bandpass regions, so we chose the refractive index of the silver layers as $n_{2}=\sqrt{\epsilon_{r}}=i k_{2}$. The refractive index of the air layers is described by $n_{1}$, and these layers have a thickness denoted by $a$. The black squares are the limit solutions to Eqs. (11) and (12) of [10], which respectively describe a cosh and a sinh field function in the metal layers matching to a cos standing wave function in the dielectric layers.

$$
\begin{aligned}
& n_{1} \tan \left(n_{1} k \frac{a}{2}\right)=k_{2} \tanh \left(k_{2} k \frac{b}{2}\right) . \\
& n_{1} \tan \left(\begin{array}{r}
a \\
n_{1} k \frac{a}{2}
\end{array}\right)=k_{2} \operatorname{coth}\left(k_{2} k \frac{b}{2}\right) .
\end{aligned}
$$

The incident and transmission materials are also air, and the structure is illuminated at normal incidence.

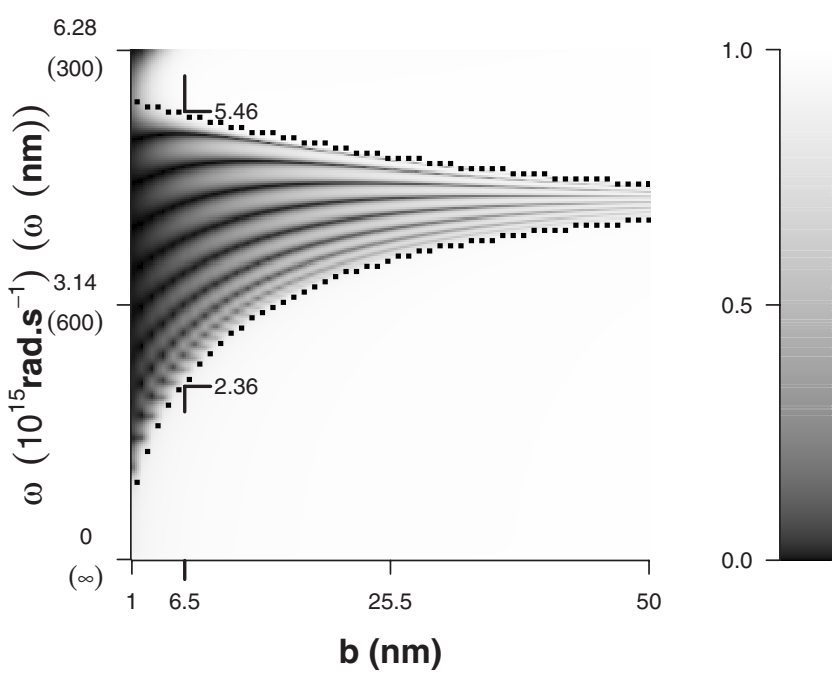

Fig. 9. Reflection efficiency response of the multilayer structure, comprising ten silver layers of thickness $1 \mathrm{~nm} \leqslant b \leqslant 50 \mathrm{~nm}$, separated by nine $166 \mathrm{~nm}$ air layers. The black squares are the limit solutions to Eqs. (2) and (3). The incident and transmission materials are also air, and the structure is illuminated at normal incidence. The frequency range is the same as in Fig. 7.
The frequency range is $0 \times 10^{15} \mathrm{rad} \cdot \mathrm{s}^{-1}<\omega \leqslant 6.28$ $\times 10^{15} \mathrm{rad} \cdot \mathrm{s}^{-1}(\infty>\lambda \geqslant 300 \mathrm{~nm})$. The reflectivity response is modelled using a multilayer Fresnel calculation.

If the ground plane of the grating is ignored, and the wires are assumed to be infinitely high, as justified for the lowest-order mode when $h>300 \mathrm{~nm}$, then it has the same parameters as the multilayer metal/dielectric stack, albeit rotated by $90^{\circ}$. However, the grating is an infinite structure in the $x$-direction, whereas the metal/dielectric stack only consists of ten metal layers because it is not possible to model an infinite number of layers using a Fresnel calculation. For the multilayer stack, the slight deviation of the band edges of the reflectivity response from the exact solutions (black squares), which occurs at low $b$ (=fd), is due to the modelled metal/dielectric structure being finite; therefore, the standing waves in the cavities and fields in the metal layers are not able to form standing fields with exactly $\cos (h)$ or $\sin (h)$ distribution functions.

From Fig. 8 it can be seen that the low-frequency limit at $2 k_{x} / k_{g}=0$ occurs at $\omega \approx 2.35$, and the higher-frequency limit at $2 k_{x} / k_{g}=1$ occurs at $\omega \approx 4.96$. These two limits are very close to the limit solutions, shown in Fig. 9, which, at $\mathrm{fd}(=b)=6.5 \mathrm{~nm}$, are $\omega=2.36 \times 10^{15} \mathrm{rad} \cdot \mathrm{s}^{-1}$ and $\omega=5.46$ $\times 10^{15} \mathrm{rad} \cdot \mathrm{s}^{-1}$. It is hypothesized, therefore, that the two limits observed in Fig. 8, for a rectangular grating with a large amplitude, are equivalent to the two band edges observed in Fig. 9, for a multilayer stack. To confirm this hypothesis, Figs. 10(a) and 10(b) present the equivalent investigation as Fig. 9 (varying the width of the metal layers-wires in this case) at the appropriate values of in plane momentum, $2 k_{x} / k_{g}=0,1$, respectively.

In Fig. 10(a) the lowest pole represents the behavior of the new, large-grating-height mode. The black squares are the solution to Eq. (2). Clearly, the dependency of the pole on the width of the wires behaves in the same way as the low-frequency band edge of a metal/dielectric stack depends on the thickness of the metal layers. In Fig. 10(b) it is clear which pole relates to the higher-frequency limit, as the other poles all behave in a different manner because the grating height is not large enough for these higher-order standing waves to have evolved into the new mode. Similarly to the low-frequency limit, this pole responds to varying wire width in the same way as the high-frequency band edge of a multilayer stack.

However, neither the low- nor the high-frequency limits seen in the grating structure match the solutions from Eqs. (2) and (3) exactly. The discrepancy between the high-frequency solution for the multilayer stack and that seen from the grating structure [Fig. 10(b)] can be understood simply as a result of the fact that the solution is occuring in the nonradiative region $\left(2 k_{x} / k_{g}=1\right)$. This fact informs us that the wavevector must have an imaginary vertical component such that $k_{y}=i k_{y}$. As $k_{0}^{2}=k_{x}^{2}+k_{y}^{2}$, the imaginary wavevector results in a negative $k_{y}^{2}$, and the frequency of the resonance is lower than expected.

The discrepancy between the low-frequency solution and that seen from the grating structure [Fig. 10(a)] can be understood in a similar manner to the discrepancy of the high-frequency solution, in that it is also the result of an imaginary vertical component of the wavevector. However, the solution occurs in the radiative region, and so 

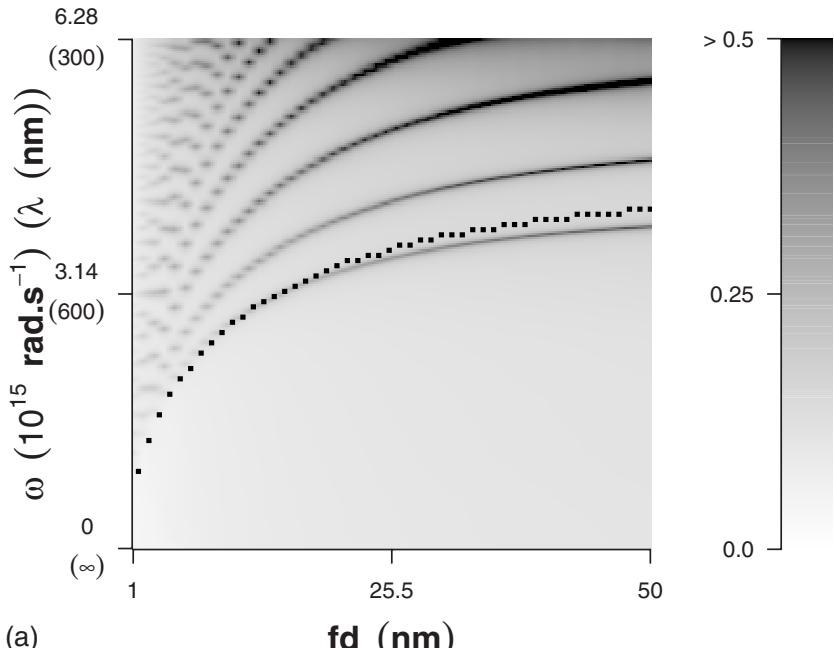

(a)

fd $(\mathrm{nm})$
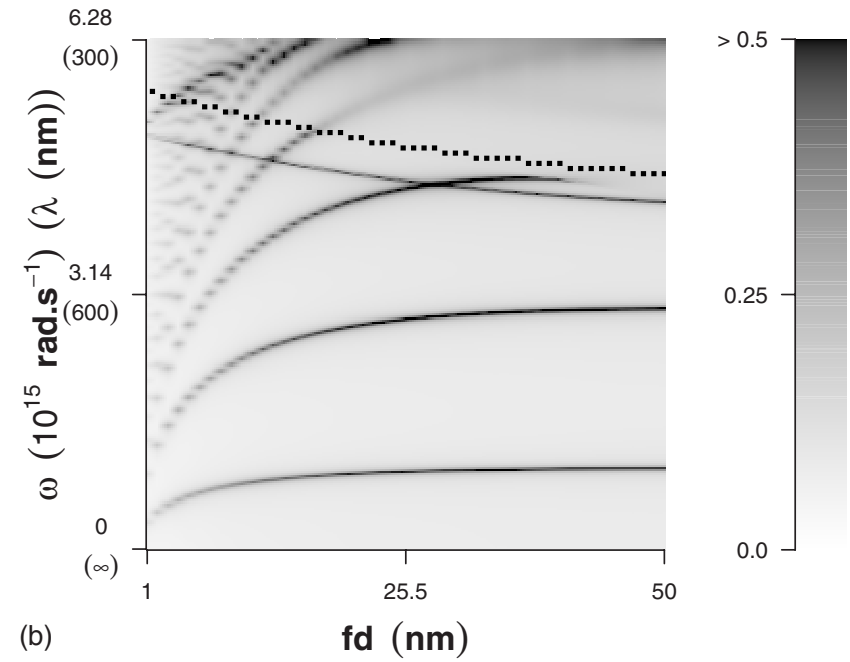

Fig. 10. Poles of the scattering matrix of a silver rectangular grating as a function of both the incident frequency and wire width for the two extreme values of $2 k_{x} / k_{g}$. The fixed grating parameters are $(1-f) d=166 \mathrm{~nm}$ and $h=320 \mathrm{~nm}$. The frequency and wire width ranges are the same as in Fig. 9. The black squares are the limit solutions to Eqs.(2) and (3).

the cause of the imaginary wavevector is due to a finite evanescent decay of the incident fields in the vertical direction. The height at which the standing-wave modes have evolved into the multilayer modes gives information about the decay length of the fields in the vertical direction. For example, if Fig. 6 is repeated for the structure in Fig. 10(a), with the ridge width set at $f d=50 \mathrm{~nm}$, then the lowest-frequency mode has converged to the limit frequency at a grating height of approximately $h_{\mathrm{lim}}$ $=225 \mathrm{~nm}$. Setting this as an approximation for the decay length of the fields allows an estimation of the magnitude of the vertical wavevector (the inverse of the decay length) as $i k_{y}=1 / h_{\text {lim }}=i 4.44 \times 10^{6} \mathrm{~m}^{-1}$. Modifying the solution of Eq. (2) by $i k_{y}$ (as above) gives a value for the expected frequency of the mode, taking into account an exponential decay of the fields within the cavities, as $\omega$ $=3.99 \times 10^{15} \mathrm{rad} \cdot \mathrm{s}^{-1}$. The actual frequency, as taken from Fig. 10(a), is $\omega=3.98 \times 10^{15} \mathrm{rad} \cdot \mathrm{s}^{-1}$. This process can be repeated for any value of $f d$, and the correct behavior is observed-the exact and modified solutions converge as $f d \rightarrow 0$ - because the decay length of the fields tends to $\infty$ (the grating height at which the modes have reached their frequency limit increases), hence $i k_{y} \rightarrow 0$.

Clearly then, the low- and high-frequency limits of the new mode presented are indeed equivalent to the low- and high-frequency band edges that occur in a multilayer metal/dielectric stack. The final question is why does only the low-frequency band edge occur at $2 k_{x} / k_{g}=0$, whereas only the high-frequency band edge occurs at $2 k_{x} / k_{g}=1$ ? Fig. 5 of [10], reproduced here in Fig. 11, answers this question.

In Fig. 11(a), the field distribution for the highfrequency band edge, the fields in adjacent cavities oscillate out of phase, while in Fig. 11(b), the field distribution for the low-frequency band edge, the fields in adjacent cavities oscillate in phase. Therefore, the periodicity of the fields that are associated with the high-frequency band edge is half the periodicity of the structure, that is $k_{x}=k_{g} / 2$, while the periodicity of the fields associated with the low-frequency band edge is the same as the periodicity of the structure, that is $k_{x}=k_{g}$. Therefore, only the
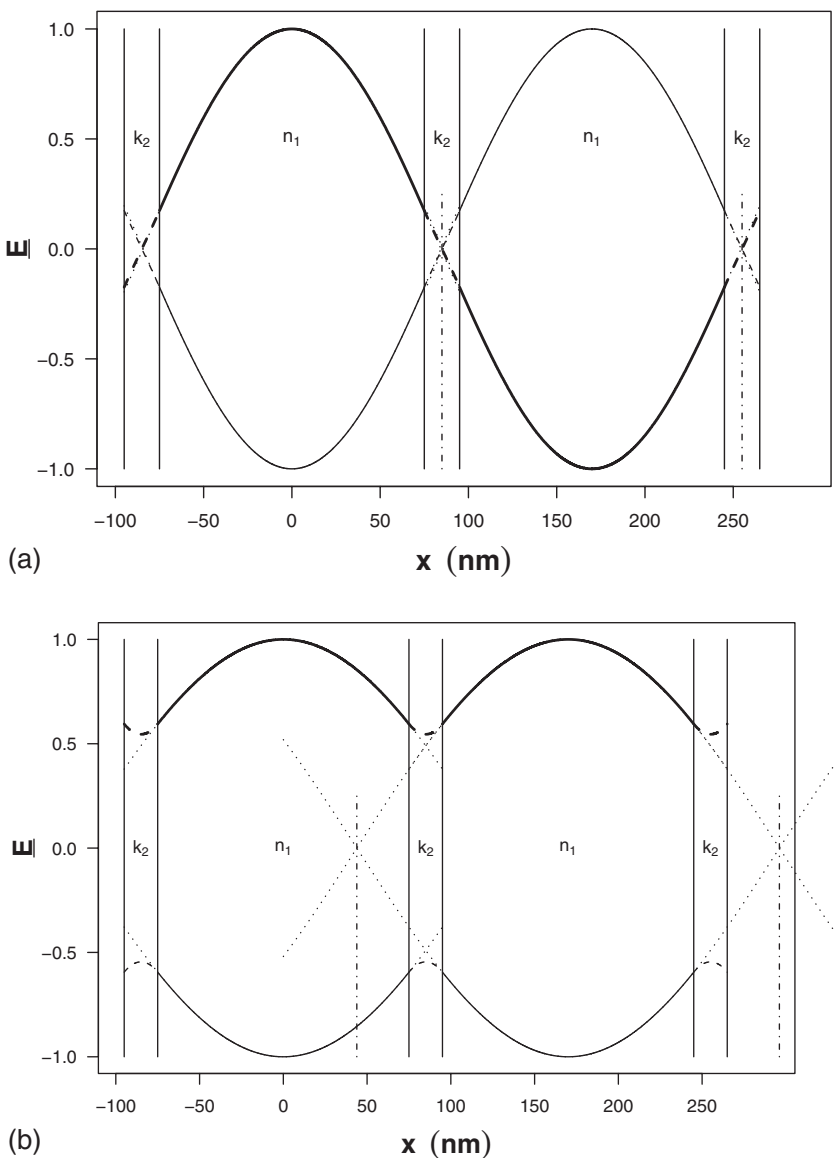

Fig. 11. The E fields for an infinite multilayer structure, comprising $20 \mathrm{~nm}$ silver layers separated by $150 \mathrm{~nm}$ air layers. The permittivity of the silver layers are approximated by the Drude model, using the parameters defined in the main text, with the imaginary part removed. The fields are plotted using the solutions to Eqs. (2) and (3), corresponding to the band edges of the bandpass region. The solid lines are the cos standing waves in the dielectric, the dashed curves are the (a) sinh and (b) cosh waves in the metal, and the dotted curves show where the dielectric standing waves would continue if no metal were present. The bold and narrow curves are $\pi$ out of phase and the vertical dotdash lines indicate the effective cavity length. 
high-frequency edge may exist at $2 k_{x} / k_{g}=1$ and may only occur for grazing incidence as expected, while the lowfrequency band edge occurs for $k_{x} / k_{g}=1$ and thus can only occur at $2 k_{x} / k_{g}=0$ as a result of a $k_{g}$ scattering.

\section{CONCLUSIONS}

The electromagnetic response of silver, rectangular surface relief gratings with a large amplitude and narrow wires has been explored. By analyzing the dispersion curves of the system for varying grating heights, it has been shown that at moderate grating heights a family of resonance exists that has much the same character as vertical standing waves in the grooves of the structure, i.e., cavity modes. These well-known cavity resonances have been shown to form from the deformation of the surface plasmon dispersion curve as the grating height is increased, due to self-coupling of surface plasmons on either vertical surface across the dielectric in the groove. It has been shown that the new family of modes presented here, which occur when the wires are narrow, evolve in a very similar manner, except that they are standing waves formed by the coupling of surface plasmons oscillating along the vertical surfaces, through the metal wires. However, these new vertical standing waves cannot be true standing waves as they do not show $1 / h$ behavior for all large $h$, because they tend to a finite low-frequency limit as $h \rightarrow \infty$.

It is shown that for very large $h$ a second new mode is formed, which is independent of $h$, by further evolution of the vertical standing waves. By comparing the behavior of this new mode with that of infinite multilayer metal/ dielectric structures, it is shown that this second new mode is equivalent to the resonant states that may exist on such a multilayer structure. Therefore, it is shown that the severe modification of the surface plasmon dispersion curves, due to large grating heights and very thin wires, results in the diffractive coupling to the same resonances that occur on multilayer structures illuminated at normal incidence. This allows the possibility of diffractive coupling to these multilayer resonances when there is an effectively infinite number of layers-something not possible in either theory or practice using planar multilayer structures.

This equivalence is not surprising, because the theory of multilayer stacks states that it is the matching of evanescent fields inside the metal layers, with standing waves in the dielectic layers, which determine the resonances the structure can support. It was mentioned in the Introduction that previous studies have also observed similar bandpass regions on multilayer structures illuminated in an ATR configuration, thereby exciting surface plasmons on the first interface that couple through the structure $[14,15]$. The equivalence presented here, between the resonances formed by simple illumination of multilayer stacks and the surface plasmon resonances on narrow wire (and large height) gratings, proves that the resonances formed on multilayer stacks illuminated in the ATR configuration are also equivalent to the resonances formed by simple illumination of such multilayer stacks.

\section{ACKNOWLEDGMENTS}

The authors acknowledge the Engineering and Physical Sciences Research Council (EPSRC) and Sharp Laboratories Europe for their financial support.

\section{REFERENCES}

1. R. W. Wood, "On a remarkable case of uneven distribution of light in a diffraction grating spectrum," Philos. Mag. 4, 396-400 (1902).

2. U. Fano, "The theory of anomalous diffraction gratings and of quasi-stationary waves on metallic surfaces (Sommerfeld's waves)," J. Opt. Soc. Am. 31, 213-222 (1941).

3. H. Raether, Surface Plasmons on Smooth and Rough Surfaces (Springer-Verlag, 1988).

4. M. B. Sobnack, W. C. Tan, N. P. Wanstall, T. W. Preist, and J. R. Sambles, "Stationary surface plasmons on a zeroorder metal grating," Phys. Rev. Lett. 80, 5667-5670 (1998).

5. W.-C. Tan, T. W. Preist, J. R. Sambles, and N. P. Wanstall, "Flat surface-plasmon-polariton bands and resonant optical absorption on short-pitch metal gratings," Phys. Rev. B 59, 12661-12666 (1999).

6. I. R. Hooper and J. R. Sambles, "Dispersion of surface plasmon polaritons on short-pitch metal gratings," Phys. Rev. B 65, 165432 (2002).

7. I. R. Hooper and J. R. Sambles, "Surface plasmon polaritons on narrow-ridged short-pitch metal gratings," Phys. Rev. B 66, 205408 (2002).

8. I. R. Hooper and J. R. Sambles, "Surface plasmon polaritons on narrow-ridged short-pitch metal gratings in the conical mount," J. Opt. Soc. Am. A 20, 836-843 (2003).

9. M. Scalora, M. J. Bloemer, and C. M. Bowden, "Laminated photonic band structures with high conductivity and high transparency: Metals under a new light," Opt. Photonics News 10, 23-27 (1998).

10. M. R. Gadsdon, J. Parsons, and J. R. Sambles, "Electromagnetic resonances of a multilayer metaldielectric stack," J. Opt. Soc. Am. B 26, 734-742 (2009).

11. J. M. Bendickson, J. P. Dowling, and M. Scalora, "Analytic expressions for the electromagnetic mode density in finite, one-dimensional, photonic band-gap structures," Phys. Rev. E 53, 4107-4121 (1996).

12. M. C. Larciprete, C. Sibilia, S. Paolini, and M. Bertolotti, "Accessing the optical limiting properties of metallodielectric photonic band gap structures," J. Appl. Phys. 93, 5013-5017 (2003)

13. M. Scalora, G. D’Aguanno, N. Mattiucci, M. J. Bloemer, D. de Ceglia, M. Centini, A. Mandatori, C. Sibilia, N. Akozbek, M. G. Cappeddu, M. Fowler, and J. W. Haus, "Negative refraction and sub-wavelength focusing in the visible range using transparent metallo-dielectric stacks," Opt. Express 15, 508-523 (2007).

14. A. Bichri, J. Lafait, and H. Welsch, "Visible and infrared optical properties of $\mathrm{Ag} / \mathrm{SiO} 2$ multilayers: radiative virtual modes and coupling effects," J. Phys.: Condens. Matter 5, $7361-7374$ (1993).

15. A. Bichri, J. Lafait, H. Welsch, and M. Abd-Lefdil, "Characterization of Berreman modes in metal/dielectric and multilayers," J. Phys.: Condens. Matter 9, 6523-6532 (1997).

16. M. G. Moharam, E. B. Grann, D. A. Pommet, and T. K. Gaylord, "Formulation for stable and efficient implementation of the rigorous coupled-wave analysis of binary gratings,” J. Opt. Soc. Am. A 12, 1068-1076 (1995).

17. L. Li, "Formulation and comparison of two recursive matrix algorithms for modeling layered diffraction gratings," J. Opt. Soc. Am. A 13, 1024-1035 (1996).

18. D. Nash and J. R. Sambles, "Surface plasmon -polariton study of the optical dielectric function of silver," J. Mod. Opt. 43, 81-91 (1996).

19. J. P. Kottmann, O. J. F. Martin, D. R. Smith, and S. 
Schultz, "Plasmon resonances of silver nanowires with a nonregular cross section," Phys. Rev. B 64, 235402 (2001).

20. Y. Takakura, "Optical resonance in a narrow slit in a thick metallic screen," Phys. Rev. Lett. 86, 5601-5603 (2001).

21. J. R. Suckling, A. P. Hibbins, M. J. Lockyear, T. W. Preist, J. R. Sambles, and C. R. Lawrence, "Finite conductance governs the resonance transmission of thin metal slits at microwave frequencies," Phys. Rev. Lett. 92, 147401 (2004).

22. M. R. Gadsdon, I. R. Hooper, and J. R. Sambles, "Optical resonances on sub-wavelength silver lamellar gratings," Opt. Express 16, 22003-22028 (2008).

23. E. N. Economou, "Surface plasmons in thin films," Phys. Rev. 182, 539-554 (1969).

24. J. J. Burke, G. I. Stegeman, and T. Tamir, "Surfacepolariton-like waves guided by thin, lossy metal films," Phys. Rev. B 33, 5186-5201 (1986).
25. D. Sarid, "Long-range surface-plasma waves on very thin metal films," Phys. Rev. Lett. 47, 1927-1930 (1981).

26. J. B. Pendry, L. Martin-Moreno, and F. J. Garcia-Vidal, "Mimicking surface plasmons with structured surfaces," Science 305, 847-848 (2004)

27. F. J. Garcia-Vidal, L. Martin-Moreno, and J. B. Pendry, "Surfaces with holes in them: new plasmonic metamaterials," J. Opt. A, Pure Appl. Opt. 7, S97-S101 (2005).

28. A. P. Hibbins, E. Hendry, M. J. Lockyear, and J. R. Sambles, "Prism coupling to 'designer' surface plasmons," Opt. Express 16, 20441-20447 (2008).

29. M. J. Lockyear, A. P. Hibbins, and J. R. Sambles, "Microwave surface-plasmon-like modes on thin metamaterials," Phys. Rev. Lett. 102, 073901 (2009). 